\title{
Mineração
}

\section{A influência do pH na reologia de polpas de caulim}

\section{(The influence of $\mathrm{pH}$ on the rheology of kaolin pulps)}

\author{
Fabíola Oliveira da Cunha \\ Engenheira Química, MSc, DSc., Departamento de Ciência dos Materiais e Metalurgia - Pontifícia Católica do \\ Rio de Janeiro, Brasil -E-mail: fabcunha@dcmm.puc-rio.br \\ Mauricio Leonardo Torem \\ Engenheiro Metalúrgico, MSc., DSc., Professor Associado - Departamento de Ciência dos Materiais e \\ Metalurgia. Pontifícia Católica do Rio de Janeiro, Brasil -E-mail: torem@dcmm.puc-rio.br \\ José Carlos D'Abreu \\ Engenheiro Metalúrgico, MSc., DSc., Professor Associado - Departamento de Ciência dos Materiais e \\ Metalurgia.Pontifícia Católica do Rio de Janeiro, Brasil -E-mail: dabreu@dcmm.puc-rio.br
}

\section{Resumo}

Polpas concentradas de caulim são amplamente utilizadas na indústria química. Algumas aplicações da polpa exigem altas taxas de cisalhamento, que, associadas às altas viscosidades de polpas concentradas, provocam dificuldades na sua utilização como cobertura. Para minimizar esse efeito, ajusta-se o $\mathrm{pH}$ e adicionam-se agentes dispersantes à polpa. O presente trabalho teve por finalidade analisar a influência do $\mathrm{pH}$ na polpa do caulim Coat 90 produzido na região do Prado/BA, contribuindo para a melhor compreensão dos mecanismos de interação solvente/mineral na reologia de polpas através dos fundamentos da química coloidal. Visando a alcançar esses objetivos, foram efetuados ensaios de potencial zeta e reológicos; foi aplicada a teoria DLVO clássica aos resultados obtidos. A análise dos dados revelou que as polpas possuem comportamento tixotrópico em valores de $\mathrm{pH}$ correspondentes a meios neutros e /ou alcalinos; a viscosidade aparente das polpas, em uma mesma taxa de cisalhamento e em um mesmo intervalo de tempo, primeiramente apresentam decréscimo com o aumento do $\mathrm{pH}$ e, posteriormente, a partir de determinado valor de $\mathrm{pH}$, há a sua estabilização; por outro lado, existe um valor de energia total de interação, a partir do qual um grau máximo de estabilidade /dispersão é conferido à polpa.

\begin{abstract}
Concentrated kaolin pulps are utilized as coatings in the chemical industry. During pulp application, high shear rates are obtained. Elevated shear rates associated to high viscosities incite difficulties on the lamina movement generating risks, bubbles or even tearing the paper. To minimize this effect $\mathrm{pH}$ adjustment and addition of dispersant agents to the pulp are made. The aim of this work was to study the colloidal behavior of the Coat 90 Prado region (Bahia State) kaolin pulps, through zeta potential and rheological evaluations combined with the classical DLVO theory. The data analysis showed that the pulps presented thixotropic behaviour and that the apparent pulp viscosity, at the same shear rates and time intervals, first reduced with $\mathrm{pH}$ increasing and then stablized at a particular constant $\mathrm{pH}$ value. On the other hand, there is a total interaction energy value which provides a maximum degree of stability/dispersion to the pulp.
\end{abstract}

Keywords: Kaolin, surface chemistry, DLVO theory.

Palavras-chave: Caulim, química de superfície, teoria DLVO. 


\section{Introdução}

Caulins são argilo-minerais, isto é, rochas constituídas de material argiloso, com baixo teor de ferro, de cor branca e cuja composição química genérica é de um silicato de alumínio hidratado: $\mathrm{Al}_{2} \mathrm{Si}_{2} \mathrm{O}_{5}(\mathrm{OH})_{4}$ (Murray \& Keller, 1993). Essa estrutura química lhe confere, entre outras, as seguintes características: inércia química,alvura, capacidade para cobertura , baixa condutividade térmica e elétrica, sendo pouco abrasivo e de baixo custo (Luz \& Chaves, 2000; Carvalho \& Almeida, 1997; Prasad et al., 1991). Tantas características tecnológicas fazem do caulim um bem mineral com vasto campo de aplicação industrial. A indústria de papel é a principal consumidora de caulim, contribuindo com cerca de $45 \%$ do mercado mundial (Luz \& Chaves, 2000). Na indústria papeleira, o caulim possui duas finalidades principais: carga e cobertura. No caso de ser utilizado como cobertura, a aplicação do caulim é efetuada sobre o papel na forma de polpas concentradas com 70\% de sólidos (em massa) (Pettendorfer, 1999). Polpas com elevadas concentrações fornecem elevados valores de viscosidade. Durante a aplicação da polpa, na produção de papel, são alcançadas altas taxas de cisalhamento, que, associadas a altas viscosidades, provocam dificuldades no movimento da lâmina, acarretando riscos, bolhas ou até mesmo rasgando o papel (Fardim, 2002). A fim de minimizar esse tipo de dano, fazem-se necessários o ajuste do $\mathrm{pH}$ e a adição de agentes dispersantes, que atuam reduzindo a viscosidade até valores adequados ao processo.

O comportamento reológico de suspensões minerais concentradas, tais como as de caulim, está relacionado às características físicas da partícula, por exemplo, formato, tamanho e distribuição de tamanho das partículas (Ortega et al., 1997a), e à estabilidade da suspensão, quando as partículas são de tamanho coloidal (Hunter, 1981). As características da partícula podem ser conhecidas a partir da sua caracterização tecnológica, onde o caulim é uma rocha de morfologia pseudo-hexagonal e com distribuição de tamanho de partículas de ordem coloidal (Murray \& Keller, 1993). Com relação à estabilidade da suspensão, temos que polpas estáveis são alcançadas pela repulsão entre as partículas minerais. Essa repulsão pode ser conseguida a partir do entendimento e da regulação da rede de forças de interação interparticulares existentes (Johnson et al., 2000). A Teoria DLVO Clássica, que fundamenta, quantitativamente, o balanço entre a energia de atração de van der Waals e a energia de repulsão eletrostática (forças de interação interparticulares) em função da distância entre as partículas, é utilizada para prever a estabilidade de uma suspensão (Laskowski \& Ralston, 1992; Hunter, 1993).

O presente trabalho teve, como principal finalidade, explicar a reologia das suspensões concentradas de caulim sob o efeito do $\mathrm{pH}$ em função dos tipos de interação interparticular existentes, utilizando-se da Teoria DLVO Clássica como meio para evidenciar os mecanismos/fenômenos envolvidos nesse processo.

\section{Materiais e métodos}

Foi utilizada, como matéria-prima uma amostra beneficiada (comercial) de caulim da Mineração Monte Pascoal Ltda. denominada Caulim Coat 90.

Inicialmente, a amostra foi caracterizada tecnologicamente. Nessa etapa, foi identificado o mineral predominante por difratometria de raios $\mathrm{X}$, foi determinada a distribuição de tamanho de partículas em sedígrafo; foi confirmada a sua morfologia em um Microscópio Eletrônico de Varredura (MEV) e foi determinada a sua alvura ISO em um Color Touch 2.

Na segunda etapa do trabalho, um estudo do potencial de superfície do caulim foi efetuado. Foram obtidos os potenciais zeta dos caulins em ampla faixa de $\mathrm{pH}$. Posteriormente, ensaios reológicos foram efetuados, onde, principalmente, a viscosidade aparente das polpas concentradas de caulim em diversos valores de $\mathrm{pH}$ foi obtida.

De posse dos dados de potencial zeta obtidos experimentalmente e de pa- râmetros adquiridos da literatura, foi efetuado o cálculo da energia de interação total, a partir da Teoria DLVO Clássica, para cada valor de $\mathrm{pH}$.

Finalmente, as curvas calculadas a partir da Teoria DLVO foram comparadas aos dados de viscosidade aparente encontrados, objetivando a explicação dos mecanismos envolvidos no processo.

\section{Resultados e discussões}

\subsection{Caracterização tecnológica}

A difratometria de raios X (DRX) é a principal técnica utilizada na identificação mineralógica das amostras de caulim. O difratograma encontrado evidencia que a amostra é constituída, essencialmente, por caulinita. Wilson et al. (1998) estudaram a mineralogia dos caulins brasileiros, concluindo que os depósitos do nordeste brasileiro, em virtude de sua origem geológica, são, essencialmente, cauliníticos, razão pela qual não foram encontrados haloisita ou diquita.

O índice de alvura é considerado uma propriedade física muito importante, pois, quanto maior for a alvura de uma amostra, maior será o seu valor agregado. O índice de alvura ISO encontrado para a amostra analisada foi $88,8 \%$, estando, portanto, dentro dos padrões exigidos pela indústria de papel: alvuras superiores a $84,5 \%$ para que o caulim seja utilizado como cobertura (Luz \& Lima, 1991).

A distribuição granulométrica do caulim Coat 90 possui cerca de $80 \%$ das partículas com tamanho inferior a $2 \mathrm{~mm}$ e aproximadamente, 34\% das partículas com tamanho menor que 0,2 mm. Esses valores caracterizam distribuições bimodais (Carvalho, 1998). Esses dados geram importantes informações: primeiro, destaca-se que pequenos diâmetros médios de partícula proporcionam maiores áreas superficiais específicas, onde maiores áreas necessitam, também, de maiores quantidades de dispersante para a estabilização da polpa e, segundo, que distribuições de tamanho de partícula em 
faixas mais largas, por exemplo, bimodais, fornecem empacotamentos melhores das partículas, gerando polpas de viscosidade menor, pois menores quantidades de água são imobilizadas nos interstícios (poros) existentes entre as partículas.

A caulinita apresenta grandes variações na morfologia e tamanho dos grãos. Essas variações podem ser associadas à origem do depósito e ao grau de cristalinidade do mineral. No caso particular da indústria de papel, a morfologia tem particular importância, porque é o formato das partículas que confere o grau de cobertura, de opacidade, de substituição das fibras celulósicas e de brilho da folha de papel produzida. A morfologia das partículas da amostra de caulim Coat 90 foi obtida a partir de fotomicrografias no MEV, utilizando-se elétrons secundários. Na Figura 1, tem-se a visão geral das estruturas das partículas da amostra, onde são observados cristais de formato lamelar com bordas irregulares assemelhando-se a hexágonos, isto é, placas pseudo-hexagonais típicas da caulinita.

\subsection{Propriedades eletrocinéticas}

Os caulins são argilo-minerais do grupo da caulinita, onde a própria caulinita é o mais importante desses minerais. A estrutura química da caulinita é formada pelo empilhamento regular (1:1) de lâminas de tetraedros de $\mathrm{SiO}_{4}$ (óxido de silício) intercaladas com lâminas de octaedros de $\mathrm{Al}_{2}(\mathrm{OH})_{6}$ (hidróxido de alumínio), ligadas entre si através de um oxigênio comum, resultando em uma estrutura fortemente polar (Murray \& Keller, 1993; Luz \& Chaves, 2000).

A carga superficial da caulinita resulta da existência de dois tipos de cargas: permanentes e não-permanentes. As cargas permanentes são produto da substituição isomórfica do $\mathrm{Si}(\mathrm{IV})$ pelo $\mathrm{Al}(\mathrm{III})$ nas posições tetraédricas do cristal e que resultam, unicamente, em cargas negativas, enquanto as cargas nãopermanentes são conseqüência de reações que ocorrem entre os grupamentos superficiais ionizáveis localizados, tan-

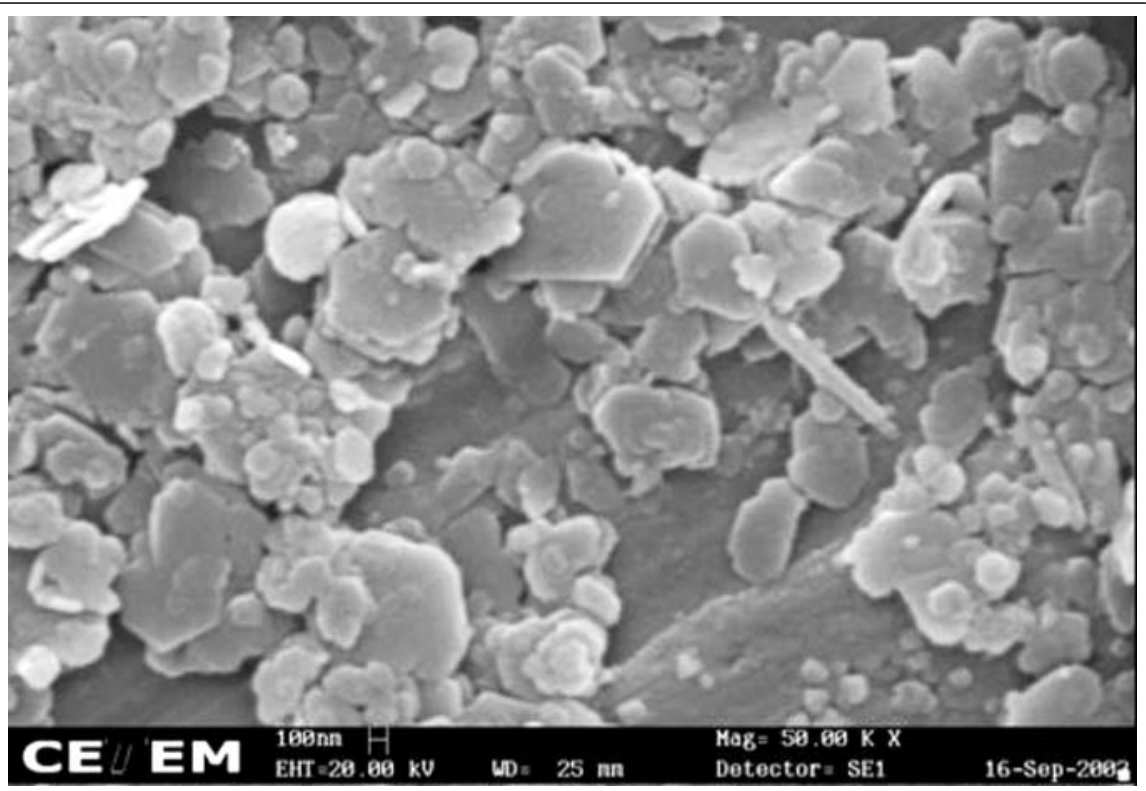

Figura 1 - Fotomicrografia obtida no MEV: visão geral das partículas de caulim Coat 90 com aumento de 50.000x.

to nas laterais, quanto na base das partículas e os íons presentes na solução aquosa. Ao ser dissociada, a caulinita forma os complexos silanol (>SiOH) e aluminol (>AlOH). O grupamento silanol somente sofre, deprotonação, contribuindo com a formação de complexos aniônicos (negativos). O grupamento aluminol é anfótero, isto é, sofre tanto protonação a baixos pHs, formando complexos catiônicos, quanto deprotonação em pHs altos, formando complexos aniônicos (Huertas et al., 1998).

Quando são utilizadas soluções de eletrólitos indiferentes em uma mesma concentração (força iônica constante), não ocorre adsorção específica, ocorrendo somente as reações de protonação e deprotonação, podendo, ainda, ser observada a variação do potencial zeta com o pH das partículas, onde as curvas encontradas são a resultante das cargas produzidas pelos complexos superficiais. As reações apresentadas a seguir mostram a protonação e a deprotonação da caulinita:

$$
\begin{aligned}
& \mathrm{AlOH}_{2}^{+}+\mathrm{OH} \rightarrow \mathrm{AlOH}+\mathrm{H}_{2} \mathrm{O} \\
& \mathrm{AlOH}+\mathrm{OH}^{-} \rightarrow \mathrm{AlO}^{-}+\mathrm{H}_{2} \mathrm{O} \\
& \mathrm{SiOH}+\mathrm{OH}^{-} \rightarrow \mathrm{SiO}^{-}+\mathrm{H}_{2} \mathrm{O}
\end{aligned}
$$

Para se avaliar o comportamento eletrocinético de determinada partícula sólida, é necessária a identificação das espécies iônicas que exercem controle fundamental na carga superficial e no potencial de superfície de uma fase dispersa (íons determinadores de potencial) e, também, das espécies iônicas que controlam a extensão da dupla camada difusa, isto é, que não estejam envolvidas em interações específicas com a superfície (íons de eletrólitos indiferentes).

O procedimento-padrão para a determinação de um eletrólito indiferente baseia-se na construção de curvas de potencial zeta em função do $\mathrm{pH}$ em diversas concentrações do eletrólito questionado. Curvas com um mesmo ponto isoelétrico sugerem espécies iônicas indiferentes à superfície da partícula.

A Figura 2 ilustra a variação do potencial zeta das partículas de caulim Coat 90 em função do valor do pH e da concentração de $\mathrm{KCl}$. Verifica-se que as curvas passam pelo mesmo ponto isoelétrico (PIE = 3,5), que as partículas são independentes da força iônica do cloreto de potássio na faixa de concentração utilizada e que os íons $\mathrm{H}^{+}$e $\mathrm{OH}^{-}$interagem mais intensamente com a superfície de caulim que os íons $\mathrm{K}^{+}$e $\mathrm{Cl}^{-}$. Essas observações sugerem que, para esta amostra, o KCl é um eletrólito indiferente, que o PIE pode ser aproximado ao 
ponto de carga zero e que o efeito acentuado que o $\mathrm{pH}$ exerce no potencial zeta indica que $\mathrm{H}^{+}$e $\mathrm{OH}^{-}$são íons determinadores de potencial. Essa análise está de acordo com estudos já realizados, que afirmam que o KCl é um eletrólito indiferente à superfície do caulim (Carvalho, 1998) e que minerais como os óxidos e silicatos podem ter as espécies $\mathrm{H}^{+}$e $\mathrm{OH}^{-}$como íons determinadores de potencial (Hunter, 1981), em particular para a amostra em questão.

\subsection{Reologia das polpas}

Reologicamente, as polpas concentradas de caulim são fluidos não-newtonianos, mais precisamente, tratam-se de fluidos tixotrópicos. No caso do uso como cobertura, esta é uma característica essencial. Neste tipo de fluido, quando a polpa encontra-se em repouso, são formadas estruturas tridimensionais provenientes da união das partículas por forças de van der Waals (Ortega et al., 1997a). A tixotropia está ligada à energia requerida para a ruptura da estrutura original (rede tridimensional) de um fluido, em outras palavras, tixotropia é a redução (reversível) da viscosidade em função do tempo sob cisalhamento. À polpa com a rede tridimensional formada dáse o nome de gel (viscosidade máxima) e, quando a estrutura tridimensional está desfeita, chama-se o fluido de sol (viscosidade mínima). Uma polpa só é considerada tixotrópica quando a transformação gel-sol e sol-gel são reversíveis (Schramm, 2000). Quanto mais rápido e pronunciadamente a viscosidade de uma tinta diminui, maior é o valor da sua tixotropia.

A partir dos reogramas encontrados e da análise das curvas obtidas para as taxas de cisalhamento de $100 \mathrm{~s}^{-1} \mathrm{e}$ $1000 \mathrm{~s}^{-1}$ em função do tempo (Figura 3), onde se encontram expostas somente três curvas ilustrativas indo de pHs intermediários até pHs elevados, foi constatado que as polpas em menores valores de $\mathrm{pH}$, mesmo quando submetidas a altas taxas de cisalhamento, permitem a formação da rede tridimensional, já que houve o aumento da viscosidade com o tempo, evidenciando, assim, o comportamento de fluido reopético. A partir de valores de $\mathrm{pH} 7,5$, primeiro, ocorre o brusco decréscimo da viscosidade aparente em um curto espaço de tempo (poucos segundos), depois, há a estabilização deste valor com a formação de um patamar, revelando um valor mínimo de viscosidade aparente caracterizando fluidos tixotrópicos. Esse mínimo é referente à melhor arrumação/orientação das partículas. Analisando essas diferenças, observou-se que o aumento do $\mathrm{pH}$ modificou a natureza da polpa, convertendo um fluido reopético para um fluido tixotrópico.

A Figura 3 também revela que viscosidades mais baixas são atingidas com maiores valores de $\mathrm{pH}$. Com o intuito de observar melhor essa relação, foi construída a Figura 4. A partir da sua análise, pode ser observada a variação da viscosidade aparente das polpas em função do pH no instante $\mathrm{t}=280 \mathrm{~s}$, onde a figura exibe viscosidades mínimas em valores de $\mathrm{pH}$ maiores que 7,5 e, uma vez alcançado esse valor, a mesma

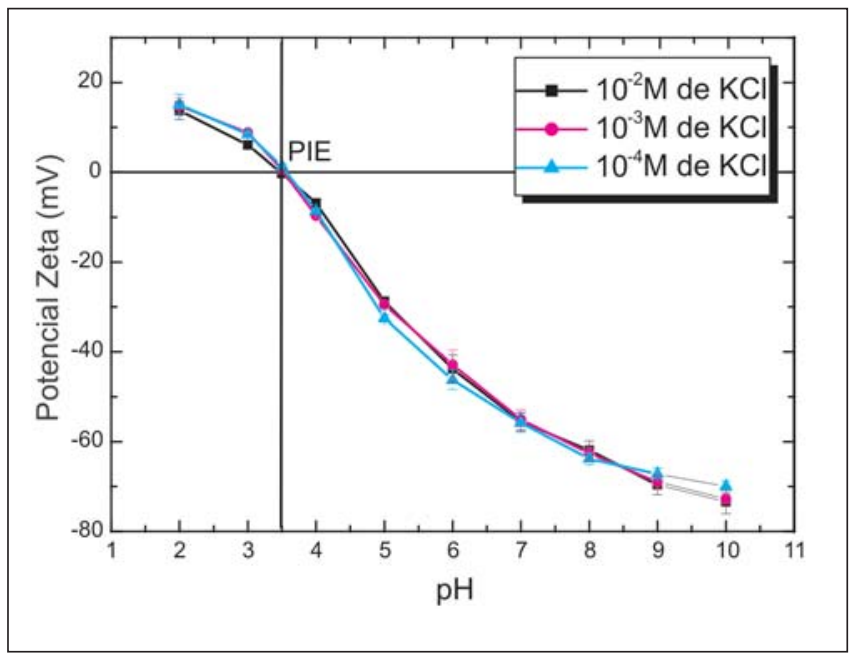

Figura 2 - Curvas de potencial zeta do caulim Coat 90 em função do $\mathrm{pH}$, parametrizado na concentração de $\mathrm{KCl}, \mathrm{PIE}=3,5$. (O PIE ou ponto isoelétrico é definido como o logaritmo negativo da atividade da espécie determinadora de potencial no plano de cisalhamento, que é correspondente ao potencial zeta nulo).

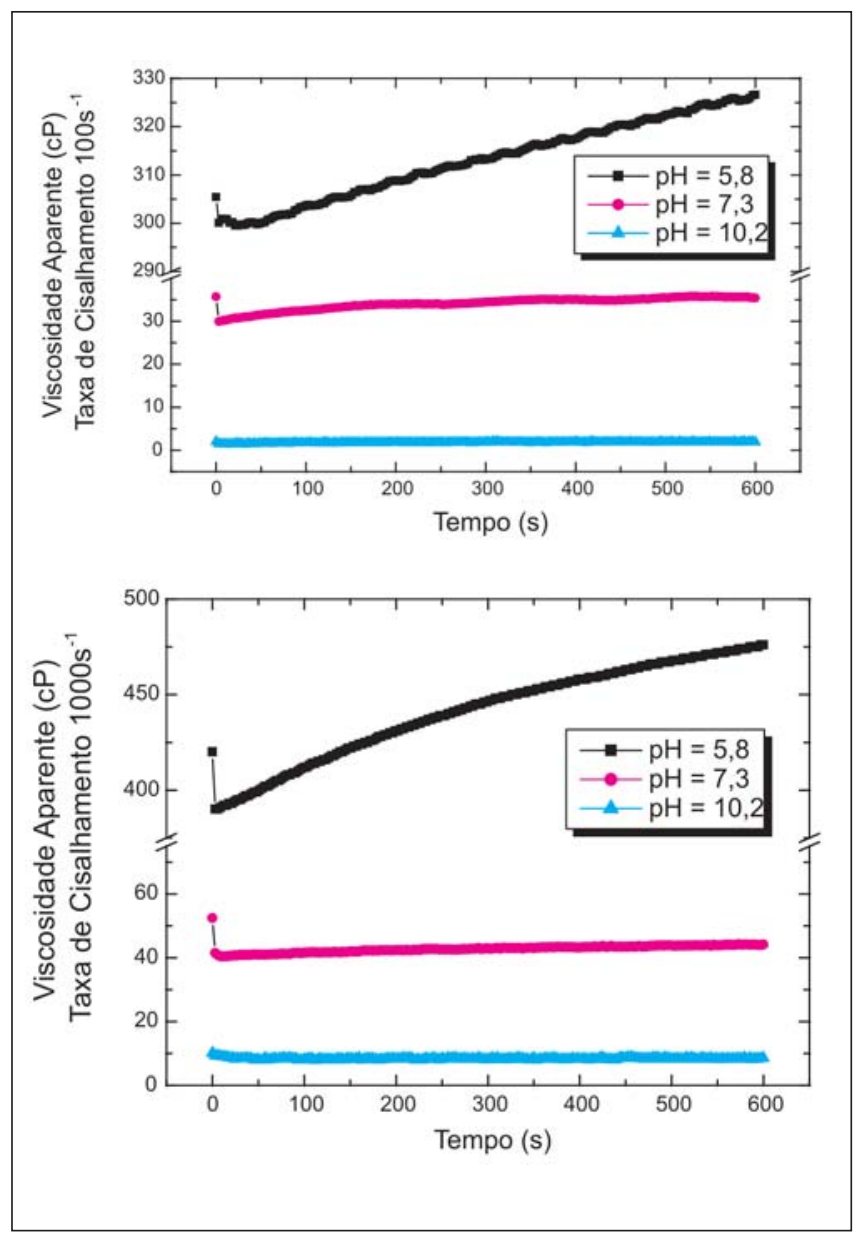

Figura 3 - Viscosidade aparente das polpas de caulim Coat 90 com $50 \%$ em peso de sólidos em função do tempo, parametrizado no $\mathrm{pH}$ e em taxas de cisalhamento constantes de $100 \mathrm{~s}^{-1}$ e $1000 \mathrm{~s}^{-1}$. 


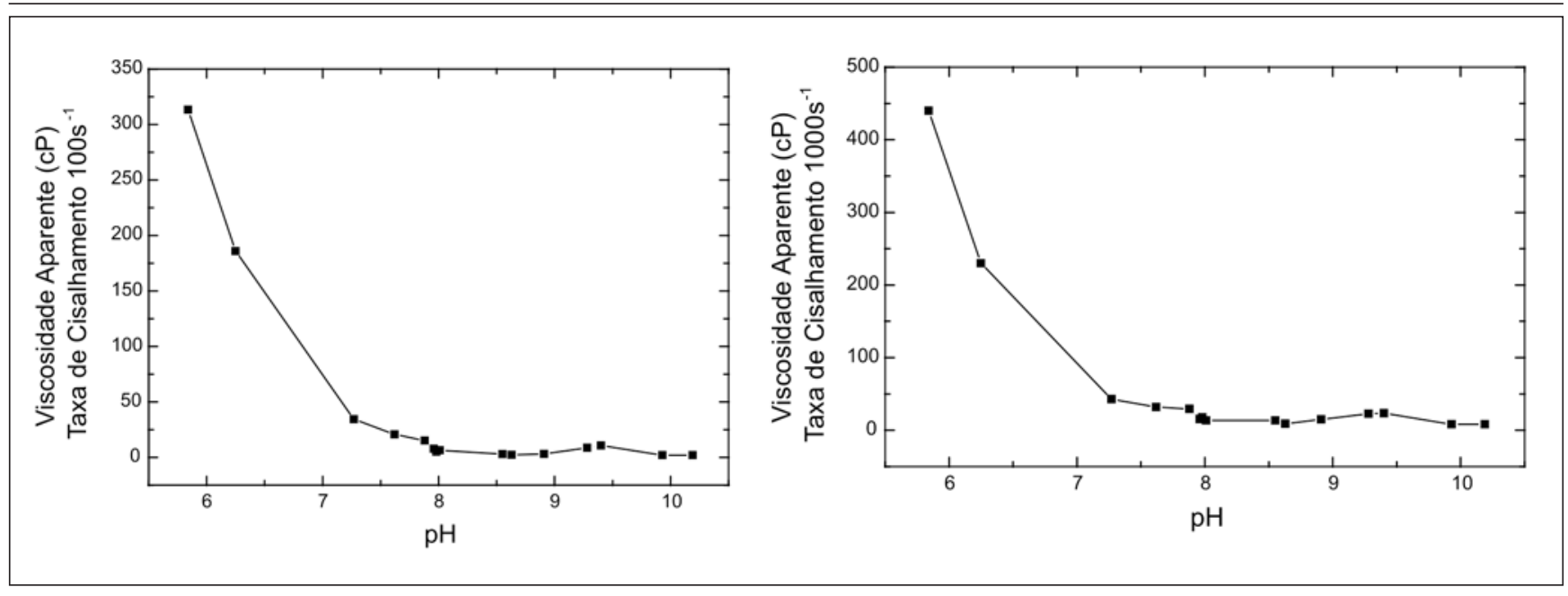

Figura 4 - Viscosidade e dinâmica aparente das polpas de caulim Coat 90 com $50 \%$ em peso de sólidos para t $=280 \mathrm{~s}$ em função do $\mathrm{pH}$ e em taxas de cisalhamento constantes de $100 \mathrm{~s}^{-1}$ e $1000 \mathrm{~s}^{-1}$.

permanece constante, indicando que o melhor arranjo tridimensional foi alcançado, isto é, as partículas encontram-se afastadas o suficiente umas das outras, de modo que a fluidez alcance o seu valor máximo.

\subsection{Aplicação da Teoria DLVO clássica}

A estabilidade de uma suspensão coloidal, de acordo com a Teoria DLVO Clássica, é determinada pelo balanço entre forças atrativas e repulsivas que as partículas sofrem ao se aproximarem. A energia de interação total pode ser alterada modificando a magnitude da repulsão. A energia de repulsão pode ser alterada pela variação da força iônica da solução (pela adição de eletrólitos) ou pela mudança do potencial de superfície das partículas (Hunter, 1981).

A formalização matemática da Teoria DLVO Clássica aqui utilizada e descrita na Equação 1 foi desenvolvida por Laskowski e Ralston (1992) e Hunter (1993) e leva em consideração partículas esféricas de mesmo tamanho onde $R>>H$.

$$
\Delta G_{I N T}=\left(\frac{64 \pi k T R \rho(\infty) \gamma^{2}}{\kappa^{2}}\right) \exp (-\kappa H)-\frac{A R}{12 H}
$$

Para eletrólitos 1:1, essa equação pode ser simplificada para:

$$
\Delta G_{I N T}=4,61 \times 10^{-11} R \gamma^{2} \exp (-\kappa H)-\frac{A R}{12 H}
$$

onde:

$\mathrm{R}=$ raio médio da partícula sólida $=2,0 \times 10^{-7} \mathrm{~m}$.

$\mathrm{A}=$ constante de Hamaker efetiva $=4,4 \times 10^{-20} \mathrm{~J}$ (James \& Williams, 1982).

$$
\kappa=0,304 / \sqrt{\rho(\infty)}(\mathrm{nm}) ; \quad \gamma=\tanh (\zeta / 103)
$$

Sendo que $\zeta$ é o potencial zeta em unidades de $\mathrm{mV}$.

De posse dessa equação, dos parâmetros retirados da literatura e dos resultados obtidos, foram efetuados cálculos pertinentes para cada polpa de caulim, a fim de se construírem gráficos de energia de interação em função da distância de separação parametrizados no $\mathrm{pH}$, com o objetivo de distinguir suspensões estáveis daquelas que favorecem a aglomeração. As energias de interação total existente entre as partículas de caulim Coat 90, em função da distância de separação, para diferentes valores de $\mathrm{pH}$, encontram-se apresentadas na Figura 5. Analisando a mesma, temos que o balanço total entre as forças atrativas de van der Waals e eletrostáticas apresentam valores de energia de interação negativos para toda distância calculada, quando se tem valores de $\mathrm{pH}$ iguais ou inferiores a 4. Nesses casos, a diminuição da distância leva, diretamente, ao mínimo primário de energia, indicando uma adesão irreversível, isto é, a suspensão coagula rapidamente. Para valores de $\mathrm{pH}$ maiores que 4, observa-se a existência de barreira energética localizada a $4 \mathrm{~nm}$, para $\mathrm{pH} 5,3 \mathrm{~nm}$, para $\mathrm{pH} 6$, e $2 \mathrm{~nm}$, para os valores de $\mathrm{pH}$ iguais ou superiores a 7. Essa barreira de energia aumenta de intensidade com o aumento do valor do $\mathrm{pH}$ e representa a energia que deverá ser ultrapassada para que ocorra a adesão irreversível no mínimo primário.

A fim de correlacionar os dados obtidos experimentalmente com os calculados pela Teoria DLVO Clássica, foi elaborada a Figura 6. Observando a mesma, temos que energias superiores a $400 \mathrm{kT}$ são suficientes para repelir e manter as partículas de caulim afastadas, conferindo o mais alto grau de estabilidade. Valores de energia de interação inferiores a esse, somente produzem suspensões metaestáveis, onde ainda é possível a agregação reversível no mínimo secundário. 


\section{Conclusões}

Os resultados obtidos permitem concluir que: i) o cloreto de potássio é um eletrólito indiferente à superfície do caulim utilizado e o cátion próton e o ânion hidroxila são os íons determinadores de potencial; ii) as polpas concentradas de caulim são fluidos de natureza tixotrópica em valores de $\mathrm{pH}$ corespondentes aos meios neutros e alcalinos e, dependendo da acidez do meio, podem tornar-se reopéticos; iii) o aumento do $\mathrm{pH}$ exerce efeito liquefante às polpas de caulim, pois causa o aumento da carga negativa do mineral; iv) pHs acima de 7,5 são os que fornecem menores viscosidades às suspensões de caulim; v) a aplicação da Teoria DLVO mostrou um ponto de barreira energética, aproximadamente $400 \mathrm{kT}$, onde ocorre a mudança de comportamento existente entre as suspensões estáveis (barreira energética maior que 400kT) e metaestáveis (barreira energética menor que $400 \mathrm{kT}$ ) e vi) a Teoria DLVO explicou, qualitativamente, o efeito do $\mathrm{pH}$ nas suspensões de caulim utilizadas exibindo um valor de energia de interação total (barreira energética) mínima necessária para a máxima dispersão da polpa, o que pode ser considerado o elo de ligação entre os dados reológicos da polpa e a química de superfície das partículas de caulim em suspensão.

\section{Agradecimentos}

Os autores agradecem o apoio financeiro ou técnico dado pelas instituições PUC-Rio, CETEM-MCT e CAPES, que viabilizou a pesquisa e o desenvolvimento desse trabalho.

\section{Referências bibliográficas}

CARVALHO, E. A. Efeito de diferentes dispersantes na viscosidade de amostras de caulim destinadas à indústria de papel e de catalisadores. In: ENCONTRO NACIONAL DE TRATAMENTO DE MINÉRIO E MET. EXTRATIVA, 17 e SEMINÁRIO DE QUÍMICA DE COLÓIDES APLICADA À TEC. MINERAL, 1. Anais..., Águas de São Pedro, 1998, p. 573-584.

CARVALHO, E. A., ALMEIDA, S. L. M. Caulim e carbonato de cálcio: competição na indústria de papel. Rio de Janeiro: CETEM/CNPq, 1997. n. 41. (Série Estudos e Documentos).

FARDIM, P. Papel e química de superfície: Parte II - revestimento e printabilidade. O Papel. p. 75-83, maio, 2002.

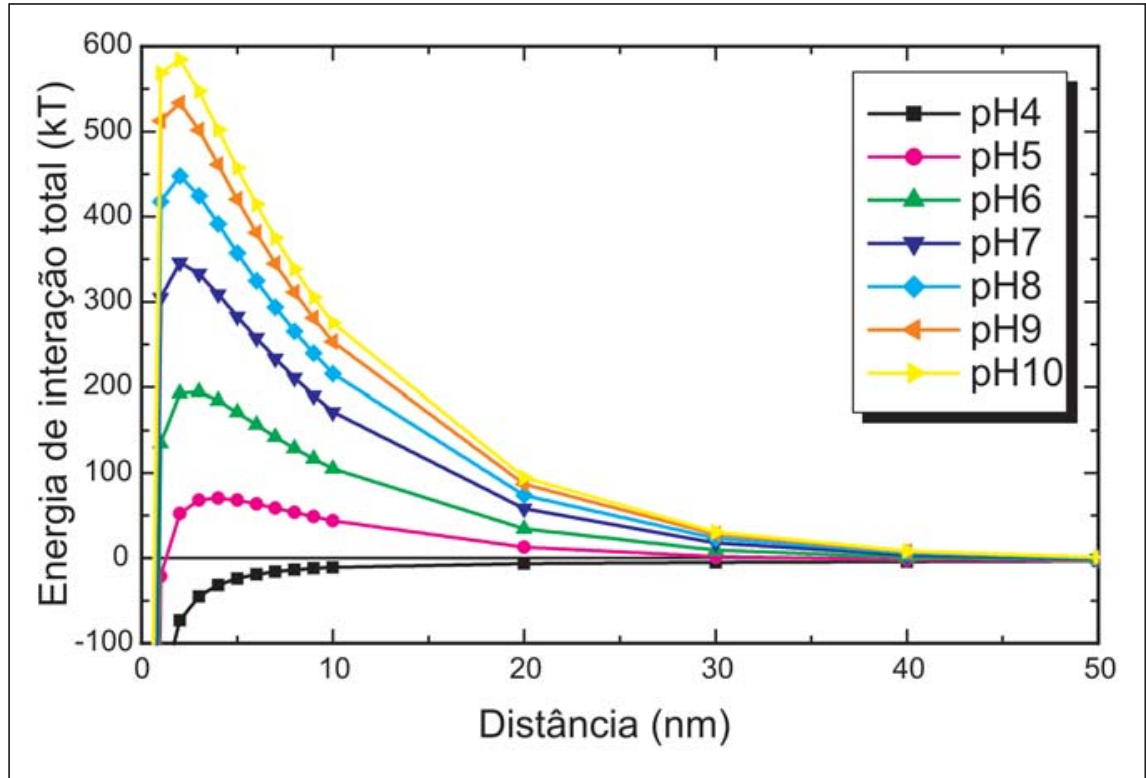

Figura 5 - Energia de interação total entre partículas de caulim Coat 90 em $1 \mathrm{mM}$ de $\mathrm{KCl}$ em função de distância de separação interparticular, parametrizado no pH.

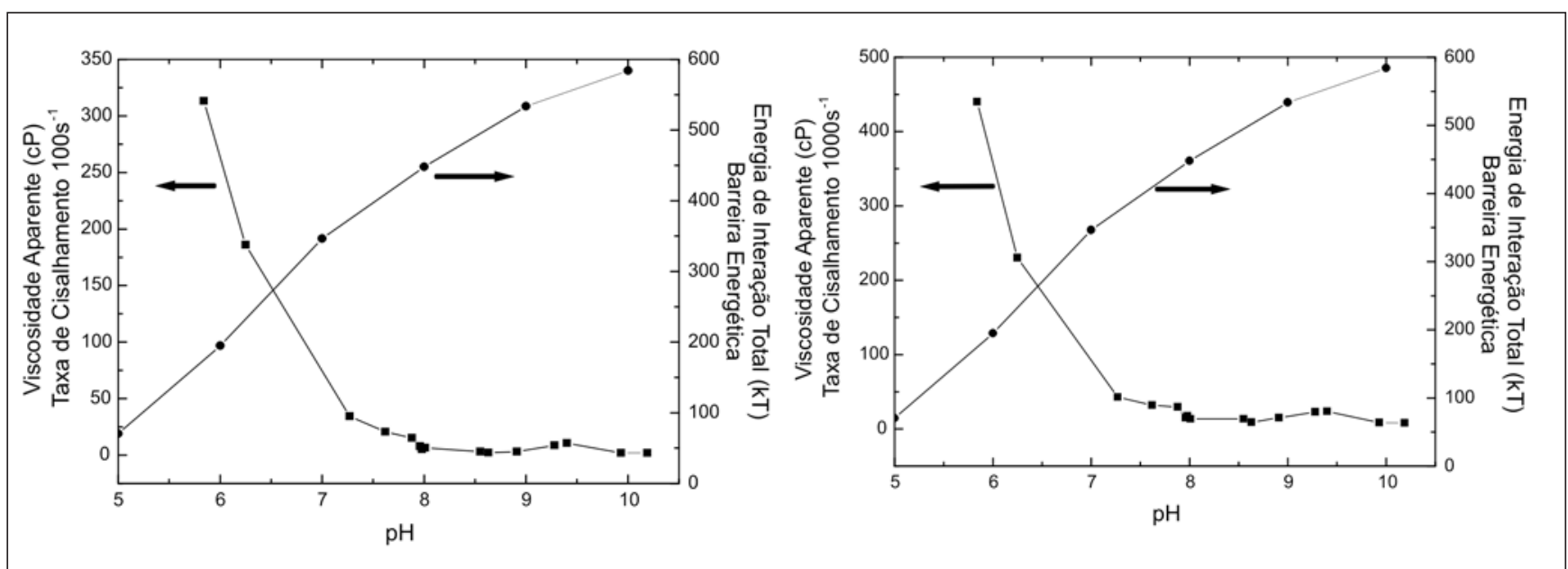

Figura 6 - Correlação entre: i) viscosidade aparente das polpas de caulim Coat 90 com $50 \%$ em peso de sólidos para t $=280 \mathrm{~s}$ e taxa de cisalhamento $=100 \mathrm{~s}^{-1}$ e $1000 \mathrm{~s}^{-1}$ e ii) barreira energética da energia de interação total entre partículas de caulim Coat 90 com $2 \mathrm{~nm}$ de distância de separação interparticular em $1 \mathrm{mM}$ de $\mathrm{KCl}$ usando a Teoria DLVO Clássica, em função do pH. 
Fabíola Oliveira da Cunha et al.

HUERTAS, F. J., CHOU, L. C., WOLLAST, R. Mechanism of kaolinite dissolution at room temperature and pressure: Part I: surface speciation. Geochimica et Cosmochimica Acta, v. 62, n. 3, p. 417431, fevereiro, 1998.

HUNTER, R. J. Introduction to modern colloid science. New York: Oxford University Press, 1993.

HUNTER, R. J. Zeta potencial in colloid science: principles and applications. London: Academic Press, 1981.

JAMES, A. E., WILLIAMS, D. J. A. Particle interactions and rheological effects in kaolinite suspensions. Advances in Colloid and Interface Science, v. 17, p. 219-232, 1982.

LASKOWSKI, J. S., RALSTON, J. Colloid chemistry in mineral processing. Amsterdam: Elsevier Science Publishing Company, 1992. v. 12.
LUZ, A. B., LIMA, R. M. F. Caracterização tecnológica de caulim para a indústria de papel. Rio de Janeiro: CETEM/CNPq, 1991. n. 48. (Série Tecnologia Mineral).

LUZ, A. B., CHAVES, A. P. Tecnologia do caulim: ênfase na indústria de papel. Rio de Janeiro: CETEM/CNPq, 2000. n. 01. (Série Rochas e Minerais Industriais).

MURRAY, H. H., KELLER, W. D. Kaolins, kaolins and kaolins. In: MURRAY, H. H., BUNDY, W. M., HARVEY, C. C. (ed.). Kaolin Genesis and Utilizations. Colorado: The Clay Minerals Society, 1993.

ORTEGA, F. S. et al. Aspectos da reologia e da estabilidade de suspensões cerâmicas. Parte I: fundamentos. Cerâmica, v. 43, p. 05-10, jan/fev, 1997(a).

PETTENDORFER, E. P. O efeito de dispersantes na reologia de polpas de caulim para a indústria de papel. Rio de Janeiro: Departamento de Ciência dos Materiais e Metalurgia, Pontifícia Universidade Católica do Rio de Janeiro, 1999. 92 p. (Dissertação de Mestrado).

PRASAD, M. S., REID, K. J., MURRAY, H. H. Kaolin: processing, properties and applications. Applied Clay Science, v. 6, p. 87-119, 1991.

SCHRAMM, G. A practical approach to rheology and rheometry. (2. ed.). Karlsruche: Gebrueder HAAKE GmbH, 2000.

WILSON, I. R., SANTOS, H. S., SANTOS, P. S. Caulins brasileiros: alguns aspectos da geologia e da mineralogia. Cerâmica, v. 44, p. 287-288, 1998.

Artigo recebido em 25/08/2006 e aprovado em 22/11/2006.

\section{Descubra as muitas informações da:}

\section{Geologia, Mineração, Metalurgia \& Materiais} e Engenharia Civil.
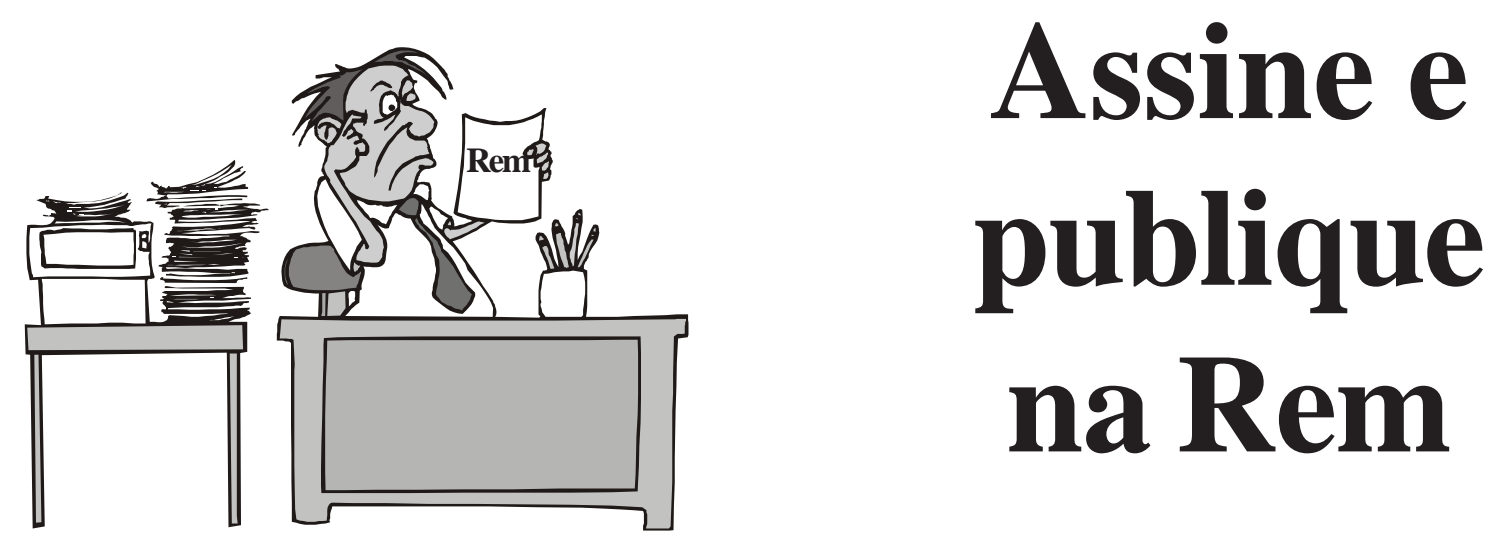

Conheça o nosso novo site: WWW.rem.com.br 